\title{
NFC-based application with educational purposes
}

\author{
Emilia Biffi \\ IRCCS E. Medea \\ Bosisio Parini (LC) \\ Italy \\ 003931877838 \\ emilia.biffi@bp.Inf.it
}

\author{
Peter Taddeo \\ IRCCS E. Medea \\ Bosisio Parini (LC) \\ Italy \\ 003931877838 \\ peterataddeo@gmail.com
}

\author{
Gianluigi Reni \\ IRCCS E. Medea \\ Bosisio Parini (LC) \\ Italy \\ 003931877350 \\ gianluigi.reni@bp.Inf.it
}

\author{
Maria Luisa Lorusso \\ IRCCS E. Medea \\ Bosisio Parini (LC) \\ Italy \\ 003931877592 \\ marialuisa.lorusso@bp.Inf.it
}

\begin{abstract}
In the past few years, technology for children and disabilities has proliferated thanks to the development of more and more inexpensive mobile technology and to the availability of freedownloadable software applications. However, at our knowledge a multisensory teaching application for both normal and disabled children still lacks in this landscape. In this work we describe the design and development of an integrated system which combines the use of a tablet, a group of toys (e.g. small plastic animals), Near Field Communication (NFC) technology and a software application with educational purposes to improve the speed of learning and entertainment value for users.
\end{abstract}

\section{Categories and Subject Descriptors}

K.4.2 [Computers and Society]: Social Issues- Assistive technologies for persons with disabilities.

\section{General Terms}

Design.

\section{Keywords}

Software application, educational, children

\section{INTRODUCTION}

The proliferation of inexpensive mobile technology is dramatically changing the landscape for both normal subjects and individuals with complex communication needs and learning difficulties. Indeed, software application, or "apps" as they are commonly called, are readily adopted by people even when a disability or a cognitive disorder is present [1].

Permission to make digital or hard copies of all or part of this work for personal or classroom use is granted without fee provided that copies are not made or distributed for profit or commercial advantage and that copies bear this notice and the full citation on the first page. To copy otherwise, to republish, to post on servers or to redistribute to lists, requires prior specific permission and/or a fee.

REHAB 2014, May 20-23, Oldenburg, Germany

Copyright $\odot 2014$ ICST 978-1-63190-011-2

DOI 10.4108/icst.pervasivehealth.2014.255350
In the past few years, programmers in the Android community have come up with varied applications for education, development, communication and rehabilitation for children with different disabilities, most notably autism [2]. Some apps have been used to improve basic speech/language capabilities as a support for traditional, impairment-based treatments. Others are available to communicate spoken, written or visual messages to other people, to facilitate communication attempts [3].

Recently, efforts have been made to use electronic media to promote healthy eating, physical activity and weight control among children [4]. Often, the goal of many developed apps is to facilitate communication between children and the outside world using pictures, applications that help form sentences, or online resources. The process has proved successful and popular among both parents and children; however, at our knowledge a multisensory teaching application for both normal and disabled or cognitively disordered children still lacks.

In this work we describe the design and development of an integrated system which combines the use of a tablet, a group of toys (e.g. small plastic animals), Near Field Communication (NFC) technology and a software application with educational purposes. In this application, each toy connected to a NFC tag, calls different activities when it passes close to the NFC reader, embedded into the tablet. These activities allow the user to read information, or a tale, or to listen to a song or to look at a picture describing that specific toy. These contents are taken from specific websites, that we filtered for children. Further, the user can choose to listen to the content of the webpages by means of a text-to-speech method. Our app reverses the standard model of communication with the outside world through pictures and text to speech functions, and uses images and audio to instruct. The application uses auditory, visual, and tactile information to improve the speed of learning and entertainment value for users. Finally, it uniquely uses NFC to incorporate physical objects outside of the tablet.

\section{MATERIALS AND METHODS}

The system integrates a tablet, equipped with a NFC reader, the custom software application and some NFC tag stickers 
(NTAG203) that are placed on hypoallergenic plastic animals, making possible the unique recognition of each toy.

\subsection{Device choice}

A Nexus 10 tablet was chosen to run the custom application. It features a Samsung Exynos 5250 system on chip, a dual-core 1.7 $\mathrm{GHz}$ Cortex A15 central processing unit and a quad-core ARMMali T604 graphics processing unit. It provides a front NFC reader, required to recognize the toys with attached NFC tags, a micro USB port, used for charging or connecting the device to a $\mathrm{PC}$ or other USB-compatible device, and a micro HDMI port to connect the system to a larger screen if necessary. The tablet's screen is large enough $(263.9 \times 177.6 \mathrm{~mm})$ for children to view images puzzles and videos clearly as well as easily pass toys over. It is widely sold and available on Amazon.com for about 260 USD. The tablet was tested to ensure it worked correctly before programming the custom education and rehabilitation software. Specifically, the Nexus 10 had the NFC tags passed over it to check if its sensor was operational, before the custom application was successfully loaded onto the tablet.

\subsection{Development of the software}

The custom application was developed by using a series of public access methods developed by Android and the android community, modifying them and integrating them into one application. The architecture of the main application is designed as follows (Figure 1). First an application to read and write onto NFC tags was created to recognize the toys as children pass them over the screen of the tablet. When a specific toy, with a recognized NFC tag is passed over the tablet a second method calls that toy's activity, a navigational page. The navigational page is unique for each NFC tag and allows the user to access a unique webview activity, that access a series of dynamic websites providing stories, interactive games and puzzles, or information. If the user wishes to access an activity with a written description in the website (e.g. information, tale), the application uses a JSOP HTML parser method to retrieve the website's text, or uses a prewritten script, and uses a text to speech method to convert the writing into speech.

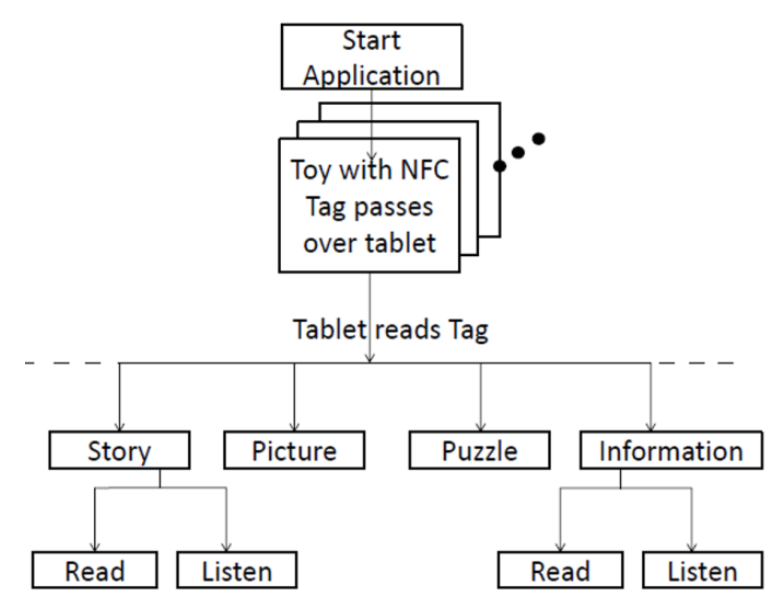

Figure 1. Architecture of the main application. A toy with a NFC tag passes over the tablet and it is recognized, thus calling different activities. These activities allow the user to read a tale (story), or to look at a picture (picture), or to make a game (puzzle), or to read didactic information (information) describing that specific toy. These contents can be read or listened by means of a text-to-speech method.

\subsection{Preliminary tests}

To evaluate the interest raised by the educational app and the usability of the integrated system, we asked 5 children with mild language disorders or delays to play with it. A structured observation grid and a questionnaire about the positive and negative reactions of the children was filled by the therapist at the end of each session.

\section{RESULTS}

\subsection{Hardware testing}

The functionality of the Nexus 10 Tablet and NFC Tags (NTAG203) was verified. A separate application, TagWriter, was downloaded onto the tablet to write plain text files onto the NFC tags to distinguish them in the Custom Application. For the current application one NFC Tag was placed below a plastic lion toy and it was programmed to contain a text file that reads "toLionActivity" to activate the lion menu, as indicated in Figure 2 .

\subsection{Custom application}

The prototype application awaits a recognized NFC tag before it displays a menu offering a puzzle activity, a story with pictures, or multimedia information associated with that NFC tag. Both the story and information pages can be read aloud, by a text to speech function that reads either text taken directly from the webpage, by a JSOUP Parser, or a predetermined script. The JSOUP parser is more dynamic and allows a larger variety of websites to be programmed into the application, however, a script can be programmed into the application to make the text to speech application sound more natural. It was determined that for the prototype the text to speech would use a script rather than the JSOUP Parser. Extra punctuation was added and some words were spelled phonetically in the script to render the speech easier to understand.

For the full application the JSOUP parsing method may be used instead because the stories and information could be read and displayed in different languages which may be phonetic and more easily fit a text to speech program.

Figure 2 displays an example of the app functionality when the plastic lion passes over the NFC reader embedded into the Nexus 10 tablet. The prototype app recognizes the NFC Tag containing the text file "toLionActivity" and prompts the application to display a menu screen. From here the user can choose to access:

- "Information" which opens one or more webpages giving scientific information about the animalhttp://www.animalfactguide.com/animalfacts/lion/, that can be read or listened by means of the text-to-speech method,

- "Story" which opens one or more websites where tales about the animal can be read (or can be listened by means of the text-to-speech method),

- "Puzzle" where games, such as puzzles can be played,

- "Picture" that returns different webpages showing pictures and photos about that specific animal. 


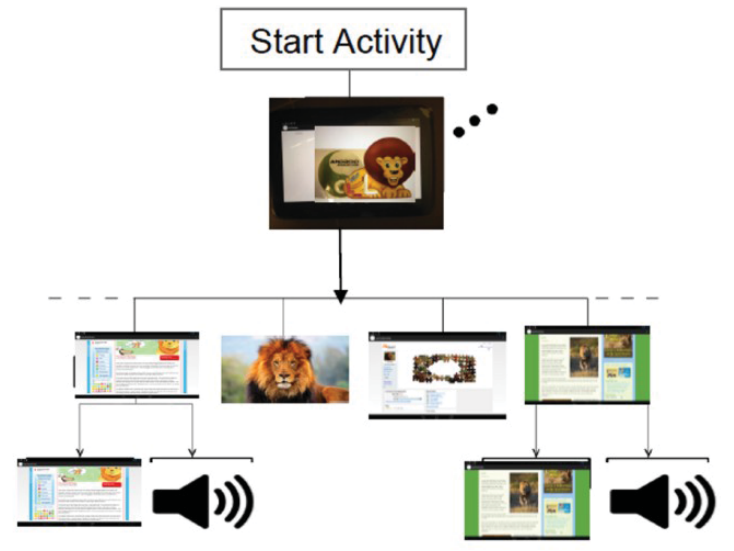

Figure 2. Architecture of the activities of the main application. A NFC tag is placed below a plastic lion toy and it is programmed to contain a text file that activates the lion menu.

Preliminary data about children's reactions and interest were collected and data are ongoing.

\section{DISCUSSION}

In the past few years, technology for children and disabilities has proliferated thanks to the development of more and more inexpensive mobile technology and to the availability of freedownloadable software applications. Apps for autism [2], speech and language disorders [1,3], and health promotion [4] are only few examples of the huge amount of software application currently available on the web. However, at our knowledge a multisensory teaching application for both normal and disabled children still lacks in this landscape. In this work we describe the design and development of an integrated system which combines the use of a tablet, a group of toys (e.g. small plastic animals), Near Field Communication (NFC) technology and a software application with educational purposes to improve the speed of learning and entertainment value for users. This integration of different elements can provide a rich spectrum of new education and research benefits. Moreover, this integrated device is not specifically designed only for disabled people and it can represent an educational tool for both able and disabled children.

A prototype app has been tested without displaying any errors: it recognizes the NFC Tag containing the text file "toLionActivity" and lets the user to choose one activity among reading/listening of information webpages, playing a puzzle, reading/listening a tale or looking at a picture. In this prototype we have a limited number of activities; however the number of activities that can be embedded into the custom software application is not limited and the system can be easily extended. Moreover, the possibility of converting a text into speech, gives the possibility of reading a website also to those children whose language and reading skills are minimal or to subjects with complex communication needs or other disabilities.

We are now developing a wider system which integrates more NFC tags, each one identifying a specific toy/object. Therefore, this app integrates more topics for the user to learn about.

Further, in the under-development app, the user's choice of one of the menu options randomly opens one of a list of predetermined websites. These lists are only limited by the number children appropriate learning websites on the internet associated with each subject, which are assumed to be increasing. This means that if the app proves successful it has the opportunity to be updated after it's been released to use a wider variety of subjects, essentially any tangible child appropriate object that can be researched, and increase the number of learning sources. The application can have a large pool of information on a topic and also provide an element of variety to keep the child's attention over a longer period. This would provide a child a tangible encyclopedia that also provided entertaining stories and games. The only two limiting factors in what can be taught is whether the subject can be represented tangibly by a toy and whether the subject is age appropriate.

Taken together this application provides a learning and educational environment with possible benefits that incorporates auditory, visual and kinesthetic processes.

\section{ACKNOWLEDGMENTS}

Our thanks to Matteo Cavalleri for his initial help and support in app development.

\section{REFERENCES}

[1] Light, J and McNaughton, D. 2011. Supporting the communication, language, and literacy development of children with complex communication needs: state of the science and future research priorities. Assist Technol. 24(1):34-44

[2] Stephan, J. and Limbrick, L. 2013. A Review of the Use of Touch-Screen Mobile Devices by People With Developmental Disabilities. J. Autism.Dev.Disord. 43, 7 (Jul. 2013).

[3] Holland, A.L.,Weinbeg, P., and Dittelman, J.2012. How to use apps clinically in the treatment of aphasia. Semin.Speech.Lang.33, 3 (Aug. 2012), 223-233.

[4] Baranowski, T., and Frankel, L. 2012. Let's get technical! Gaming and technology for weight control and health promotion in children. Child Obes. 8, 1 (Feb. 2012), 34-37. 